\title{
Bindung und Phantasie in einer psychoanalytischen Behandlung
}

\author{
Aleksandar Dimitrijevic ${ }^{1}$ (Belgrad)
}

Zusammenfassung: In der Geschichte der Psychoanalyse scheint das Konzept der Phantasie einen polarisierenden Einfluss auf die prominentesten Autoren und Schulen ausgeübt zu haben. Freud, Melanie Klein und ihre jeweiligen Nachfolger betrachteten Phantasie als ausserordentlich bedeutsam, zuweilen bedeutsamer als interpersonelle Beziehungen. Dagegen wird Phantasie in der Arbeit John Bowlbys und der Tradition der Bindungstheorie weitgehend vernachlässigt, und zwar $z u$ Gunsten der frühen Kind-Pflegeperson-Beziehung und Traumatisierungen. Ich versuche, mit der Darstellung einer fünf Jahre dauernden Psychoanalyse im liegenden Setting an diese Kontroverse anzuknüpfen. Bei dieser zeigte sich, wie frühe Vernachlässigungserfahrungen der Patientin untrennbar von ihren Bindungsmustern, sexuellen Phantasien, und der Wahl ihrer Liebesobjekte waren. Diese Arbeit führt mich zur Schlussfolgerung, dass theoretische Polemiken die klinische Realität nicht abbilden. Ein Psychoanalytiker scheint kontinuierlich sowohl in ein "Trieb-Modell» als auch in ein "Trauma-Modell»verwoben zu sein.

Schlüsselwörter: Phantasie, Trauma, Trieb, Bindung, Uebertragung

Es scheint, dass das Konzept Phantasie die PsychoanalytikerInnen während des gesamten letzten Jahrhunderts polarisiert hat und als ein der Realität entgegengesetztes Konzept aufgefasst wurde. Ein Teil der PsychoanalytikerInnen betrachtete Phantasie als fundamental und stützte ihre theoretischen Modelle auf sie ab. Dies begann in Freuds Denken 1897, aber ein noch markanteres Beispiel dafür ist die Arbeit von Melanie Klein. Dort ist es so ausgeprägt, dass man bei der Beschäftigung mit ihrem Theoretisieren nicht anders kann, als immer wieder zum Konzept der Phantasie zurückzukehren: «Es gibt mehrere Schlüsselkonzepte im Modell von Melanie Klein. Nach Klein entstehen psychische Strukturen aus einer Vielzahl innerer Objekte (Phantasien über Personen im Leben des Kindes), deren Charakter sich durch unbewusste Phantasien verändert, wenn das Kind heranwächst. Die Phantasien des Kindes verwandeln sich durch seine tatsächlichen 
Erfahrungen beim Austausch mit der Umwelt, und im Laufe der Entwicklung stellt es seine Objektwelt immer weiter in den Dienst eines inneren, primär defensiven Beziehungssystems» (Fonagy 2003: 91).

Der andere Teil der Psychoanalytiker aber hat Theorien dieser Art als simplizistisch und unwissenschaftlich abgetan, ja, es wurde gar gesagt, sie stellten einen «Angriff auf die Wahrheit» dar (Masson 1985). Einige Kritiker gingen so weit, eigene Modelle zu formulieren, die der Phantasie keine wesentliche Rolle im menschlichen Geist zugestanden. Am Wichtigsten finde ich das Beispiel der Bindungstheorie und -forschung. Es ist heute gut dokumentiert, dass ihr Gründer John Bowlby seit seiner Supervision bei Melanie Klein gegen deren Betonung der Phantasie rebellierte, als sie darauf bestand, dass er sich bei einem Kind, das er behandelte, ausserhalb der verinnerlichten Objektbeziehung und Phantasie nicht für die reale Beziehung zu dessen leiblichen Eltern interessieren dürfe (vgl. Issroff 2005: 59). So kam es, dass Mary S. D. Ainsworth in einem Interview, das sie Peter Rudnytsky gab, feststellte, die Rolle der Phantasie in der Bindungstheorie sei ziemlich absichtlich vernachlässigt worden (Rudnytsky 2000: 41). Diese Einsicht einer der engsten Mitarbeiterinnen John Bowlbys stand nicht allein. Bowlbys frühere Arbeiten kritisierend, schrieb Donald Winnicott aus persönlicher Erfahrung: «[...] Dr. Bowlby redet, als ob es so etwas wie Phantasie im frühen Säuglingsalter nicht gäbe [...]. Unbewusste Konflikte scheinen in Bowlbys Psychologie keinen Platz zu haben und er scheint dem Konzept des Verlusts und der Wiederbelebung eines Objekts in der inneren Welt des Säuglings, das heisst im psychischen Apparat, keinen Raum zu geben» (Winnicott [1959] 1989: 431). Und auf einer persönlicheren Ebene erinnert sich Judith Issroff, dass Bowlby Ausdrücke wie «Aggression» und «Phantasie» abschätzig als «Portmanteau- oder Umbrella- oder Omnibus-Worte» bezeichnete. 1966 bat Issroff Bowlby um einen Rat in einem ihrer Fälle, bei dem auch Wahnvorstellungen und Halluzinationen vorkamen. Bowlbys Antwort war, er wolle «diese Frage lieber auf Eis legen» (Issroff 2005: 56).

Seltsamerweise dauert dieser Trend bis heute an. Sowohl bei aufwändigen Themenverzeichnissen wie bei «Child Care and the Growth of Love» (Bowlby 1965) als auch bei allen drei Teilen von Bowlbys Hauptwerk «Attachment and Loss» (Bowlby 1969, 1973, 1980) kommt der Begriff Phantasie nicht vor, das gleiche gilt für beide Editionen des voluminösen «Handbook of Attachment» (Cassidy und Shaver 2008) und für die zeitgenössische Bindungs-Literatur, jedenfalls soweit ich dies überprüfen konnte. Bei der Mentalisierungsbasierten Therapie (MBT) wird ausdrücklich davor gewarnt, der Phantasie zu viel Aufmerksamkeit zu schenken. Wenn sie über ihre Arbeit mit Borderline-Patienten schreiben, machen Fonagy 
und Bateman (2008: 154) geltend, dass Ermunterungen zum aktiven Phantasieren über den Therapeuten schädliche Auswirkungen haben können. Ziemlich sicher wären sie anderer Meinung, wenn Sie über Patienten mit einer weniger schweren Pathologie schrieben. In einer etwas späteren Arbeit (Bateman, Fonagy und Allen 2009) gibt es in der Tat mehr Nuancen. Obwohl die Autoren die möglicherweise schädigendeWirkung der Übertragungsanalyse wiederholen, wird nun klar, dass sie das Nachdenken über Phantasien nicht für eine Gefahr per se halten: «Wir empfehlen Vorsicht beim Gebrauch dieser Technik in einem frühen Stadium der Therapie und/oder wenn der Patient im nicht mentalisierenden Modus funktioniert. Zudem meinen wir nicht, die Stimulation der Phantasie sei automatisch schädlich, sondern es handelt sich um eine Technik, die mit hohem Risiko verbunden ist» (op. cit.: 770). Es bleibt zu hoffen, dass MBT-Techniken für die Arbeit mit Personen, die eine weniger schwere Traumatisierungsgeschichte haben, ein anderes Verhältnis von Realitätsprüfung und Phantasiebearbeitung aufweisen.

Und um wieder zur Gegenseite zurückzukehren: Obwohl Bowlbys Werk viele brillante Forscher inspirierte und man in jeder empirischen psychoanalytischen Forschung zu den Themen Traumafolgen, Schicksal früher Bindung, Mentalisierungsstörungen oderWirksamkeit von Psychotherapie seinen Einfluss unschwer entdecken kann, anerkennen auch einige der wichtigsten Psychoanalytiker den Wert der Bindungstheorie nicht (Bergmann 2004: 342); Kernberg betrachtet sie als «grobe Simplifizierung» (ebenda: 347), Blum kritisiert, dass sexuelle und aggressive Triebe sowie unbewusste Phantasien missachtet werden (ebenda: 350), und Stepansky (2009: 68) stellt fest, dass die wichtigen Arbeiten auf diesem Gebiet nicht wirklich psychoanalytische Werke sind.

Obwohl es verlockend wäre, mögliche Gründe für diese Spaltung zu diskutieren - seien sie biographisch, historisch-politisch oder konzeptionell - so liegt dies jenseits der Reichweite dieses Artikels. Was ich stattdessen ohne jeden Anspruch auf Originalität versuche, ist zu veranschaulichen, wie äußerst unfruchtbar diese Diskussion im Bereich der klinischen Psychoanalyse ist. Das sorgfältige Anhören der freien Assoziationen ergibt immer eine Melodie ineinander verflochtener Motive bestehend aus Erfahrungen mit realen Personen, Träumen sowie Phantasien über sie (für eine bahnbrechende Arbeit über sexuelle Phantasien und vor allem über ihre Rolle bei der Überwindung von Traumata siehe Kahr 2007: 332-334). Und in der psychoanalytischen Arbeit offenbaren sexuelle Phantasien in der Mehrzahl der Fälle darunter liegende Bindungsbedürfnisse, welche in der Kindheit und/oder in der frühen Kindheit unmöglich zu erfüllen waren; sie sind nicht einfach nur Derivate biologisch verwurzelter Triebe. Der eigentliche Grund, 
glaube ich, warum Menschen die Hilfe der Psychoanalytiker suchen, liegt in ihrer Unfähigkeit, anscheinend unwiderstehliche Phantasien von den Tatsachen ihres eigenen Lebens zu trennen - eine Unfähigkeit, die durch Schmerz oder vielmehr durch Traumata verursacht wird.

Meine Lehrerin in diesem Bereich war eine Klientin, die ich Frau A. nenne, welche grosszügigerweise bereit war, ihre Träume, Phantasien, Assoziationen und Erinnerungen während den fünf Jahren ihrer immer intensiven und oft turbulenten Analyse mit mir zu teilen. Die Analyse wurde meiner Meinung nach vorzeitig abgebrochen und blieb dadurch unvollständig.

Als Frau A. das erste Mal in meine Praxis kam, war mein Eindruck, es könnte sich um ein High-School-Mädchen handeln, und ich wurde dann von ihrer Mitteilung überrascht, sie sei fast dreissig. Frau A. war seit einem halben Jahr mit einem Mann verheiratet, mit dem sie zuvor schon zwei Jahre zusammengelebt hatte. Sie beschrieb ihren Ehemann als einen Mitbewohner, in den sie nie verliebt gewesen war. Es stellte sich heraus, dass sich seit der Heirat die Haltung ihres Vaters ihr gegenüber stark verändert hatte, was ihr Hauptproblem war: Seit sie eine eigene Familie gegründet hatte, distanzierte dieser sich von ihr. Frau A. hatte ihren Vater immer als Unterstützung und Halt erlebt. Daher wurde sie sehr verunsichert, auch war ihr Ehemann nicht bereit, ihr so viel Unterstützung zu bieten, wie es ihr Vater getan hatte.

Während der ersten Wochen und Monate ihrer Analyse beeindruckten mich Frau A.s Fähigkeit, meine Interventionen zu nutzen, und ihr Interesse an TraumAnalysen. Sie zeigte dies, indem sie manchmal verkündete: «Lasst uns Träume spielen! Letzte Nacht träumte ich ...» Frau A. schien witzig und intelligent; sie investierte eine Menge an Aufwand und Mut in den Prozess und machte öfters eigene Interpretationen, welche zum Teil ausgezeichnet waren.

Ganz von Anfang an teilte Frau A. ihre zahlreichen und häufigen Phantasien mit mir, einige waren erschreckend, andere erotisch, viele zeigten auch ihr Bild von mir. Und mit «ganz von Anfang an» meine ich ihre allererste analytische Sitzung. Minuten, nachdem sie auf der Analysecouch gelandet war, erzählte mir Frau A., sie habe in der letzten Nacht geträumt, einen ihrer Kollegen geküsst zu haben. Sie wachte sofort auf, dachte dann aber sofort: «Spielt keine Rolle, es war nicht der Psychologe.» Dann erzählte sie eine Phantasie, welche sie am Sonntag beruhigt hatte: Sie war an einem Strand, entspannt, und fragte sich, welchen Mann sie neben 
sich haben möchte, «auch wenn es mein Vater wäre». Schließlich beendete sie die Sitzung mit den Worten, es sei für sie wichtig gewesen, mich bei unserem ersten Gespräch unattraktiv zu finden.

Die nächste Sitzung wurde dem Umstand gewidmet, dass jeder ihrer Kollegen ihre «Phantasie bevölkert» habe. Das heisst, Frau A. unterhielt erotische Phantasien über jeden von ihnen, hielt das aber vor ihnen geheim. Sie war noch nicht bereit, den genauen Inhalt dieser Phantasien preiszugeben. Stattdessen nutzte sie die Zeit lieber, um mir von ihrer Vergangenheit und von den wichtigsten Personen in ihrem Leben zu erzählen. Die Beschreibungen waren reich und detailliert, aber mündeten meist in die Schlussfolgerung: Man habe sie als Kind vernachlässigt, ihre Mutter sei von sich selbst besessen gewesen, und was sie selbst erreicht habe, sei allein ihrem Vater zu verdanken.

Ich erfuhr, dass Frau A.s Eltern noch Studenten waren, als sie zurWelt kam. Sie kümmerten sich während der ersten acht Monate um sie und gaben sie dann der Grossmutter mütterlicherseits in Obhut, welche in einer fernen Provinzstadt lebte. Das Nächste, woran sie sich erinnern konnte, war die Mandeloperation im Alter von drei Jahren: Sie fühlte sich am Boden zerstört, als die Krankenschwestern sie mit Gewalt von ihren Eltern trennten. Nach der Operation kam sie wieder zur Grossmutter.

Im Nachhinein glaube ich, dass ich fast sofort mit ihr sympathisiert und gehofft habe, ihr helfen zu können. Wahrscheinlich ist es genauer, zu sagen, sie verstand es meisterhaft, in mir das Bedürfnis zu wecken, sie zu pflegen und ihr jene Sorge und jenes Verständnis entgegenzubringen, welche ihre frühen Entbehrungen kompensieren könnten. Auch schien es mir, als verstünde ich sie sehr gut: ihre somatischen Symptome, ihre Partnerwahl, den ersten Traum, den sie hatte, als sie noch in Psychotherapie war. In diesem Traum war sie in meinem Praxisraum, während ich in allen vier Ecken sass und sie beobachtete. Sie näherte sich jeder der vier Repliken von «mir» und streute blaue Bonbons auf die Köpfe. Nach dem Traum sagte sie als Erstes, dass es sich bei den blauen Bonbons um eine blaue Pille gegen Angstzustände gehandelt habe, die sie eingenommen habe, noch bevor sie zur Psychotherapie gekommen sei. Sie erinnerte sich daran, dass die Lieblingsbonbons ihrer Kindheit auch blau gewesen waren. Wir erforschten den Traum weiter und als ich vorschlug, dass sie bereit sei, das anzubieten, was sie am meisten liebe, um die Prüfer zu beruhigen, stimmte sie zu, denn andernfalls würden sie etwas Faules in ihr entdecken, sagte sie. Diese innere Verkommenheit war ein häufiges Thema der kommenden Monate, und wir suchten es auf verschiedene Art und Weise anzugehen. 
Wenn Frau A. ihre Mutter beschrieb, konnte aber nichts Übles im Bild entdeckt werden. Sie erlebte ihre Mutter als in einer Phantasie- und Kunstwelt lebend, mit manchmal überdramatisierten Gefühlen; auch als eine kokette Frau, welche sich von ihrem Vater scheiden liess, als sie zwölf Jahre alt war, um bald darauf einen neun Jahre jüngeren Mann zu heiraten. Frau A. gab an, sie sei nach der Scheidung ihrer Eltern für ihren Vater und ihren jüngeren Bruder zur zentralen weiblichen Figur avanciert. Folge war, dass ihre weiblichen Identifikationen und die Anstrengungen zur Entwicklung ihrer malerischen Fertigkeiten auf der Strecke blieben.

Diese erste Ebene der Einführung fand ihre Fortsetzung mit der Information, dass auch Frau A.s Vater mehrere Jahre später wieder heiratete, als sie bereits Studentin war. Fast sofort, geriet sie in schwere Konflikte mit ihrer Stiefmutter, mit dem Resultat, dass ihr Vater sich entschied, Frau A. eine andere Wohnung zu kaufen. Sie empfand dies als Ausweisung aus derWohnung, in der sie aufgewachsen war. Wie zu erwarten, stand das Erleben dieserVertreibung in Zusammenhang mit ihren frühesten Kindheitserfahrungen. Nur, diesmal wurde sie schwanger und, «um Rache an meinem Vater zu üben", beschloss sie, die Schwangerschaft abzubrechen. Dabei entdeckte sie, dass sie niemanden hatte, auf den sie sich verlassen konnte. Gemäss Erinnerung blieb sie nach dieser Prozedur eine ganze Woche im Bett, las die Bibel und fühlte sich leer.

Nach etwa drei Monaten Analyse schien Frau. A. genug Vertrauen zu haben, um mir mehr über ihre Gedanken über mich mitzuteilen. Es kann auch nur ein Zufall gewesen sein, der dazu führte, denn einmal an einem Freitag tönte sie ganz anders und zum ersten Mal bezahlte sie nicht für ihre Sitzungen. Zum Ersten brauchte ich sie nichts zu fragen, denn Frau A. erklärte mir, sie habe am Morgen ein Treffen gehabt, für welches sie sich schöner gekleidet hätte; sie trug hohe Absätze und war geschminkt. Sie räumte ein, dass sie seit der ersten Sitzung darauf bedacht war, immer die gleiche Kleidung zu tragen und unordentlich zu erscheinen. Es war leichter, während Sie auf der Couch war - «die Couch ist eine Lügnerin, es ist einfach zu betrügen, während du auf der Couch liegst» - aber als die Sitzung zu Ende war, wirkte sie völlig verwirrt und beschloss, nicht zu bezahlen und ging wortlos hinaus. Frau A. interpretierte auch das Meiste dieses Materials selbst. Sie erzählte mir, Kleidung sei eine Möglichkeit, ihr Frausein zu verstecken und sich als Mädchen auszugeben. Sie meinte, das sei eine Flucht vor dem mütterlichen Vorbild. Es kam ihr in den Sinn, dass sie als Kind einmal gesagt hatte, die Sonne sei gelb wie Mutters Haar, so schön wirkte sie auf sie. Ich sagte ihr nur, «der Eindruck, den Sie als Kind von der Schönheit ihrer Mutter hatten, ist vielleicht 
ein Lichtstrahl in ihrer Kindheit gewesen». Sie wurde dann sehr traurig und sagte, sie habe ihre Mutter innig geliebt, dass aber der Zeitpunkt kam, an dem sich alles veränderte. Unmittelbar anschliessend meinte sie: «Jetzt werde ich Ihnen alles erklären! Am Anfang war ich glücklich, dass Sie mein Therapeut werden würden. Ich wusste, dass ich mich von Ihnen nie angezogen fühlen werde und dachte, eine gleichaltrige Person könnte mich leichter verstehen. Aber dann war ich fasziniert, wie intelligent Sie sind, und es nahm mich wunder, ob ich mich je in Sie verlieben könnte. Ich würde immer gerne mit Ihnen sprechen wollen, aber ich möchte kein bisschen mehr mit Ihnen haben. Ich habe versucht, mir unser gemeinsames Leben vorzustellen. Ich sah unseren ersten Streit: Ich drehe mich zu Ihnen um und sage wütend: ‘Ich kann das nicht ertragen! Mein ganzes Leben sagen Sie mir, was ich zu denken habe!> Das ist stupid! Das würde ich meinem Vater sagen. Und ich brauche jemanden, der mir Illusionen und Begeisterung vermittelt. Und das sind Sie. Ich habe gesehen, wie Sie mich fragen: «Bist du ok? Geht es dir gut? Ihr Kopf lag an meiner Schulter. Sie waren an einem schönen Ort (es folgt Stille). So sehr ich versuche, Ihnen alles zu erzählen, schäme ich mich noch immer für gewisse Dinge. Ich kann Ihnen nicht alle Details erzählen. Dennoch wird der Moment kommen, in dem Sie alles wissen werden.»

Ich sagte nicht viel dazu, obwohl ich den Eindruck hatte, dass dies ein Zeichen von Veränderung und Hoffnung war. Auch fand ich ihre verführerischen Manöver nicht verlockend; sie wirkte eher wie ein Kind, das eine Verführerin spielt.

Hiernach begannen Frau A.s wichtigste Erfahrungen umfassender in die Übertragung hinein zu kommen. Sie berichtete über mehrere Träume, die das gleiche erotisierte Motiv hatten: In einem davon «gab es etwas Romantisches, wir sind wie zusammen, aber ich weigere mich, Sie zu küssen». In einem anderen kommt sie zwei Stunden früher zu einer Sitzung in ein riesiges Universitätsamphitheater, sehr aufgewühlt und mit der Bitte, mich zu umarmen, dann aber entzieht sie sich meinen Versuchen, sie zu küssen. Schliesslich «ein sehr schöner und leichter Traum», in welchem wir zwei uns die Hand halten. Dann war sie überrascht von Träumen, in welchen sie mit mir in einem Kellerraum eingeschlossen war. So etwas sei ihr noch nie passiert, gab sie an. Sie dachte, sie könnte sich in mich verlieben, sollte es mir gelingen, sie zu verstehen und eine Verbindung zwischen ihren Erfahrungen herzustellen. Aber sollte ich in mehreren Sitzungen nichts Gescheites sagen, wäre sie über mich und ihre Therapie enttäuscht. Und schon bald begann sie, die Analyse als Gefängnis zu erleben. Ihre Forderungen nach drei anstatt vier Sitzungen pro Woche wechselten ab mit «Sie wissen es sicher besser, ich werde Ihren Rat befol- 
gen.» Beispielsweise als ich sagte, sie bilde so intensive Bindungen, dass sie sich gefangen, eingeschränkt und verführt fühle, schien sie zuzustimmen, aber wenige Wochen später mussten wir von Neuem beginnen.

Vor meinem Sommerurlaub erzählte sie mir, dass sie während des Geschlechtsverkehrs immer verschiedene Phantasien habe, sie beginne gerne mit Phantasieren, um Lust zu erleben. Sie fühlte sich meistens als kleines Mädchen und stellte sich vor, siebzehn Jahre alt zu sein, ansonsten hätte sie Phantasien über Männer, die nicht ihr Ehemann seien: «Ich habe immer Angst, jemand könnte sehen, dass ich viele Männer auf einmal begehre. Ich habe so viele Probleme mit meinem Ehemann. Ich will unbedingt Sex während meiner Periode, aber er verweigert sich mir. (In diesem Augenblick realisiere ich, dass sie weint und während des Sprechens Tränen mit ihrer Hand abwischt.) Er verwendet jedes Mal ein Kondom. Er denkt, dass ihm dies hilft, die Ejakulation hinauszuzögern. Aber ich würde es geniessen, seinen Samen in mir zu spüren. Er tat es nur einmal und sagte, es sei abstossend für ihn. Ich phantasiere ständig, dass ich viele Männer gleichzeitig befriedigen kann. Sie sind alle verschieden. Nein, sie unterscheiden sich durch die Grösse ihrer Genitalien. Sie alle ejakulieren in mir. Das gibt mir das Gefühl, voll zu sein, gibt mir ein gutes Gefühl. Das habe ich immer gemocht.»

Ich reagierte darauf, indem ich kurz sagte, der Zweck dieser Phantasie könnte sein, ihre Gefühle von innerer Leere und Verkommenheit zu beruhigen. Dazu sagte Frau A. zuerst: «Ich denke, das stimmt.» Dann, nach einer Weile, fügte sie hinzu: «Das ist wahrscheinlich der Grund, weshalb ich das Wort «voll` wiederholt habe.» Diese Phantasie wurde zu einem der zentralen Themen in Frau A.s Analyse, aber in diesem Augenblick konnten wir kein tieferes Verständnis dafür gewinnen. Der Grund dafür war nicht überraschend: Sie reagierte sehr intensiv auf meine Sommerferien, die vier Wochen dauerten - wie wir herausfanden, zuerst ausgehend von der Angst, dass Beziehungen eine Trennung nicht überdauern; dann mit Wut und einem Hautausschlag, nachdem ich zurückgekommen war und unsere Arbeit fortsetzte.

Nach mehreren Wochen der Nachferienphase berichtete Frau A. einen Traum, in welchem sie meinen nackten Fuss während einer Sitzung sieht und ihn sanft berührt; woraufhin ich ihr im Traum antworte, dass ich mich so von ihr angezogen fühle wie sie sich von mir, dass dies aber für sie nicht gut sei. Frau A. sagte sofort, dass sie das an ihren Vater erinnere: Er hatte ein Bein-Problem und es waren seine Füsse, die ihn am meisten schmerzten. Sie hatte es immer gemocht, die nackten Füsse ihrer Freunde zu beobachten. Es war ihr schon immer wichtig, dass ihr Vater keine Schmerzen hatte. Ich sagte ihr, mir scheine, sie würde lieber selber 
Schmerzen akzeptieren oder etwas, das ihr lieb sei, aufgeben, wenn sie dadurch ihren Vater vor Schmerzen bewahren könne. Das war der Moment, in dem mir Frau A. zum ersten Mal erzählte, dass ihr Vater so wütend und hartnäckig sein konnte, dass sie sich ihm unterwerfen und einfach tue, was er von ihr verlangte.

Wenige Tage später kam Frau A. sehr aufgeregt in die Sitzung. Sie begann, über eine Beziehung zu sprechen, die sie vor mehr als zehn Jahren hatte, und es war offensichtlich, dass ihre Erinnerungen schmerzhaft waren. Sie war noch ein High-School-Mädchen, als sie einen fast zwanzig Jahre älteren Mann traf, vor dem sie sich so fürchtete, dass sie ihm kaum in die Augen sehen konnte. Als sie das erste Mal allein waren, befahl er ihr, sich auszuziehen, und sie tat es, da sie nicht in der Lage war, zu protestieren. Frau A. hielt es für eine Missbrauchsbeziehung und hatte immer noch Angst, dass er sie mit HIV angesteckt haben könnte. Damals sei sie völlig verrückt gewesen, sagte sie mir. Der Krieg war ausgebrochen und ihre Schulfreunde wurden in die Armee eingezogen, und einige von ihnen fielen in den Kämpfen. Sie hoffte, dieser Mann sei in der Lage, sie zu beschützen, da er durch seine Autorität Respekt und Angst einflösste. Auch waren ihre Eltern nicht mehr in der Lage, ihr Schutz zu bieten. Frau A. räumte ein, sie habe nie geglaubt, zu wagen, mir dies zu erzählen. Sie befürchtete, dass ich jeden Funken Respekt vor ihr verlieren würde, «falls Sie überhaupt welchen hatten», wenn ich erführe, wie tief sie gefallen war. Aber an diesem Morgen hatte sie den Namen dieses Mannes in den Nachrichten gehört - er war vor dem Haager Tribunal wegen Verbrechen gegen die Menschlichkeit angeklagt worden. Frau A. war am Boden zerstört. «Ich habe mich von einem Kriegsverbrecher ficken lassen und suchte seinen Schutz! Ich bin der schlimmstmögliche Abschaum», schrie sie, in Tränen aufgelöst.

Ich hatte den Eindruck, dass dies die erste Runde unsere Arbeit abschloss. Wir haben die Themen Bindung, Sexualität, Traumata und Schutz erforscht, welche meiner Meinung nach absolut untrennbar von einander waren und in ihren Phantasien gipfelten: Das Verlassenwerden, das Frau A. in der frühesten Kindheit erlebt hatte, führte zur Sorge um die Bindungen, wo ihr Gefühl der Leere zu verwirrtem sexuellen Verlangen führte mit dem Bedürfnis nach Schutz und sklavischer Unterwerfung unter Männer, die in der Regel älter waren als sie, und die sie als mächtig erlebte. In der nächsten Runde arbeiteten wir an den gleichen Problemen, sprachen häufig über die gleichen Erfahrungen und Erinnerungen, während die Übertragung zu einem gewissen Grad abkühlte und Frau A.s Einsichten tiefer und leichter erträglich wurden.

Jedes Mal, wenn Frau A. verreiste, wurde ich für sie plötzlich perfekt - «der Bestaussehendste, Zärtlichste, Gescheiteste, Liebste». Sie begann, öfter als sonst 
über mich zu phantasieren, aber sie hielt das für normal, «weil Sie meine Eltern und viele andere wichtige Personen symbolisieren». Frau A. war noch nicht bereit, etwas über den Inhalt ihrer mich betreffenden sexuellen Phantasien zu berichten, denn es war zu wichtig für sie, diese Phantasien als ihr Eigenes zu betrachten, mich in der Phantasie bei sich zu haben, obwohl sie diese Phantasien ohne zu zögern ihren Freunden beschreiben konnte.

Frau A.s Ehe verschlechterte sich allmählich und sie sagte oft, sie könne sich Sex mit vielen Männern vorstellen, nicht aber mit ihrem Ehemann. Jedes Mal, wenn sie Liebe machten, phantasierte sie, entweder viele Penisse in sich zu haben oder dass viele Männer, einer nach dem andern, mit ihr Sex haben würden. Einmal wurde der Ehemann Frau A. gegenüber gewalttätig. Eines Morgens nahm er sie an der Kehle und schrie sie an, während sie leise weinte und nicht in der Lage war, etwas zu sagen. Während der Sitzung, später am selben Tag, gestand Frau A., dass ihr Vater sie mehrfach auf die gleiche Weise gewürgt und geschlagen hatte. Sie dachte jedoch, ihr stilles Hinnehmen der Tätlichkeit ihres Mannes stehe in keinem Zusammenhang damit. Auf meine Frage hin erklärte Frau A., ihr Vater habe sie geschlagen, bis sie 20 Jahre alt gewesen sei, «aber nur, wenn ich ihm nicht gehorchte oder zu spät nach Hause kam». Am nächsten Tag erzählte mir Frau A., sie fühle sich ihrem Ehemann sehr viel näher und begehre ihn zum ersten Mal wieder seit zwei Jahren.

Eines Tages erzählte mir Frau A., dass sie ihre Mutter gebeten habe, ihr zu berichten, wie sie als Kind gewesen sei. Die Erinnerungen der Mutter begannen mit dem Geständnis: «Wir wollten dich nicht, du bist uns passiert». Frau A.s Grossmutter mütterlicherseits bat das junge, noch unverheiratete Paar, die Schwangerschaft nicht abzubrechen und versprach, sich um das Kind zu kümmern - und Frau A. wurde ihr vor dem ersten Geburtstag in Obhut gegeben. Dieses Gespräch hatte natürlich katastrophale Folgen für Frau A. In den kommenden Monaten wiederholte sie immer wieder, niemand habe sie je gewollt und es wäre besser gewesen, sie wäre gar nicht geboren worden. Sorgfältig begann ich, ihre Aufmerksamkeit auf das noch bestehende Gefühl, abgetrieben zu werden, zu richten: Sie erlebte einen Teil ihrer selbst als tot und verdorben; sie brauchte viele Männer, um sich voll zu fühlen; sie fühlte sich unsicher und unbeschützt.

Die sexuellen Phantasien waren jetzt auf ein Gefühl von Emanzipation von mir bezogen. Sie fühlte sich leer und langweilig. Sie hatte das Gefühl, Besserung bedeute das Ende der Therapie und Trennung, was neue Ängste, weitere Phantasien und Frustration auslöste, weil es nicht möglich war, mit mir ausserhalb des analytischen Rahmens die Beziehung zu vertiefen. 
Frau A. hatte oft den Eindruck, es wäre für sie sehr wichtig, mich beschnuppern zu können. Sie möchte, sagte sie, mein Hund sein, mir absolut treu sein und mich gleichzeitig so kennen lernen, wie dies Welpen untereinander tun, von gleich zu gleich. Beschnuppern, dachte sie, war wie Berühren, aber nicht im üblichen Sinne, sondern als beste Möglichkeit, herauszufinden, ob sie mich wirklich mochte oder nicht. Wir verbrachten viel Zeit mit dem Durcharbeiten der Verbindung zwischen Treue, Überlegenheit und Sexualität, und sie kam zum Schluss, sie habe Sexualität und Schutz verwechselt: Im Glauben, leer und langweilig zu sein, tauschte sie Sex für Schutz ein. Ihre sexuellen Phantasien, viele Männer zu befriedigen, traten wieder auf, aber diesmal hatte sie den Eindruck, dass diese Männer sie idealisieren würden: Ein einzelner Mann konnte ihr nicht genügen.

Es wurde Frau A. bewusst, dass ihre Weiblichkeit vereitelt wurde, weil sie befürchtete, ihr Vater könnte denken, sie sei gleich geworden wie ihre Mutter. Ihre Kleider und Manieren wurden eleganter und sie wirkte nun wie eine Geschäftsfrau. Ihre Introspektionsfähigkeit und ihr Wagemut waren bemerkenswert und ich glaubte, wir näherten uns dem Ende der Analyse.

Ich war überrascht, als die Sitzungen erneut dem Mann galten, den sie den «Kriegsverbrecher» nannte, und dabei weinte sie sich in der Regel ihre Augen aus. Frau A. sagte, sie werde sich diese Beziehung nie verzeihen. Sie fuhr fort, ihr erster Geschlechtsverkehr sei praktisch eine Vergewaltigung gewesen, und dieser Mann habe sie einmal zu Oralsex gezwungen, während sein Bruder dabei zusah. Sie erklärte, dass sie schreckliche Angst vor ihm gehabt habe, sich dann aber einredete, in ihn verliebt zu sein und nicht ohne ihn leben zu können. Sie verstand ihr Verhalten so, dass sie in dieser Zeit mehr als alles andere Schutz brauchte und er die einzige Person war, die diesen bieten konnte.

Frau A. wechselte die Arbeitsstelle - sie war anspruchsvoller und besser bezahlt - und innerhalb von einigen Monaten begann sie eine Affäre mit einem Kollegen, der sowohl älter als auch ihrVorgesetzter war. Von Anfang an idealisierte sie seine Fähigkeiten und seine Erfahrung. Auf einer eher psychologischen Ebene faszinierte er sie, weil er fast absolute Kontrolle über seine Gefühle hatte, wozu sie ihrer Überzeugung nach nie fähig gewesen sei. Ich hatte immer mehr das Gefühl, die Analyse verarme und alles werde auf diese Affäre «übertragen». Ich versuchte sie weiterhin zu erreichen und fragte sie wieder einmal, während sie die neue Affäre beschrieb, ob sie denke, dass sie eher wie eine Frau klang, die durch einen Mann fasziniert ist, oder wie ein Kind, das von einem Erwachsenen fasziniert ist. Nach einer Schweigeminute antwortete sie: «Als Kind. Ich bin immer so. Immer ein Kind. Und Ihre Wiederholungen werden dies nicht ändern. (Sie schweigt dann 
erneut.) Aber es gibt auch eine Frau. Ich denke schon. Ich habe immer sexuelle Phantasien über diese Männer. Über jene, die faszinierend sind. Ich habe immer jeden von ihnen gesehen, - ich sollte wohl besser sagen ‘von Euch», Sie waren einer von ihnen - ich sah jeden bei jedem Geschlechtsverkehr $25 \mathrm{Mal}$ in mich eindringen. Ich weiss, was das heisst. Denn dann werden sie mich voll machen, weil ich mich immer leer gefühlt habe. (Wieder ein Moment Stille.) Es bedeutet auch, dass ich so erregend bin, dass sie mich $25 \mathrm{Mal}$ wollen; niemand konnte genug bekommen von mir.»

Die Liebesaffäre endete innerhalb von wenigen Monaten und hinterliess sie niedergeschlagen und rachsüchtig. Die Arbeitsstunden wurden zu einem bitteren - manchmal gnadenlosen - Machtkampf. Es kam mir vor, als artete alles, was wir in fast drei Jahren erreicht hatten, in ein riesiges Ausagieren aus. Sie begann zu trinken und schlief nach schlaflosen Nächten einige Male während der Sitzung ein. Einmal verlor sie auf der Autobahn die Kontrolle über ihren Wagen und erschrak ernsthaft darüber, dass sie hätte umkommen können. Zu Beginn der nächsten Sitzung bat sie mich, auf die Benutzung der Couch verzichten zu dürfen.

Sie trennte sich von ihrem Ehemann, aber sie befand sich in einerVerfassung, in der sie keinen Mann attraktiv fand. Einmal schlug ich vor, vielleicht mache die enge Verbindung von Liebe und Leiden in ihrem Erleben «normale» Männer unattraktiv für sie. Weshalb ihr Kollege just durch den ersten Eindruck, dass er gefährlich ist, attraktiv gewesen sei. Es kam zu folgendem Dialog:

Frau A.: «Ich weiss, ein Teil von mir will ihn und ein anderer fühlt, er ist es nicht. Ich möchte nie mit ihm leben oder einen Film ansehen und Hände halten. Er ist es nicht, aber es gibt etwas furchtbar Spannendes an ihm. Es ist nicht der Sex, das sollten Sie nicht denken. Am Anfang erregte er mich, dann merkte ich, dass der Sex schlecht war. Wir haben uns immer versteckt, fickten meistens im Büro. Ich habe es nur einmal genossen, als er mich in seine Wohnung einlud. Auch hat er Probleme. Vorzeitige Ejakulation. Er hasst mich, weil er merkt, dass er mir auch jetzt nicht widerstehen kann. Ich weiss, Sie denken, es sei Masochismus - ich leide so stark und erreiche so wenig. Und Sie haben recht. Und ich komme davon nicht weg. Wie heute. Ich hielt Distanz und sofort begann er, mich jeden Augenblick zu rufen. Er gab vor, Informationen für einen Bericht zu benötigen, aber wir beide wussten, dass er log. Er ist ein Hai, Sie wissen, ein Raubtier.»

$A D: \quad$ «Die Frage ist, glaube ich, warum Sie sich selbst mit ihrem empfindlichsten Teil einem Hai angehängt haben.» 
Frau A.: «Ich wusste, dass er gefährlich ist. Das spürte ich sofort.»

$A D: \quad$ «Und wenn ich mich recht erinnere, verliebten Sie sich kurz danach in ihn.»

Frau A.: (Schweigt etwa fünf Minuten lang.) «Ich sah eine wunderbare TV-Show. Ich weiss nicht, ob Sie sie gesehen haben. ÜberWale. Wenn ein Baby-Wal geboren wird, ist er eine leichte Beute. Insbesondere für Killerwale. Sie sind die grösste Bedrohung. Aber wenn das passiert, gibt es noch einen Weg, das Baby zu retten. Die erwachsenen Tiere bilden einen Ring um das Baby, so dass die Killerwale nicht zu ihm gelangen können.»

$A D: \quad$ «Sieht aus, als ob Sie um Schutz und Sicherheit flehen und sich gleichzeitig mit dem potenziellen Folterer einlassen.»

Frau A.: "Ja. Und wissen Sie, warum? Ich denke, es gibt zwei Gründe. Ich leide daran, etwas Besonderes sein zu müssen. Und was ist spezieller, als ein Raubtier zu zähmen? Ausserdem geniesst man einen absoluten Schutz, weil das wildeste Tier im eigenen Dienst steht.»

$A D: \quad$ «Und haben Sie je erlebt, dass dem so ist?»

Frau A.: «Nein, niemals. Das ist der Grund, weshalb ich es so unbedingt will. (Sie ist wieder still, aber nicht für lange.) Kürzlich erinnerte ich mich daran, was mein Vater mir angetan hat. Wissen Sie, wie er mich geschlagen hat? Wenn ich später heimkam, als er es mir befohlen hatte, schlug er mich nicht einfach, er schlug meinen Kopf gegen die Wand. Er fesselte meine Hände an den Radiator, damit ich erfahre, wie gross seine eigene Angst war, wenn ich zu spät kam. Glauben Sie das? Haben Sie schon je so etwas gehört? Ich habe erkannt, dass es dumm von mir war, zu sagen, er habe mich auf seine Weise geliebt, so gut er eben kann. Jetzt denke ich, dass er mich nie liebte. Und ich denke, er war nie fähig, jemanden zu lieben. Und doch weiss ich, dass ein Teil von ihm in mir wohnt und auch ich niemanden auf normale Weise lieben kann.»

In der Tat fühlte sie sich extrem allein und isoliert - von ihrer Familie, ihren Freunden und Kollegen, von mir und ihrer Therapie. Sie sagte mir mehrmals, dass sie es vorziehe, alleine zu sein und zu phantasieren, als zu ihren Sitzungen zu 
kommen. Sie hatte den Eindruck, dass ihre Phantasien in der Therapie «übersetzt» wurden, und wenn immer das passierte, verschwanden ihreWünsche und sie fühlte sich leer zurückgelassen. Die Phantasien seien ihr zu teuer, um sie zu besprechen, sie möchte sie nicht verlieren, sagte sie. Ich versuchte, ihr mitzuteilen, was ich davon halte, nämlich dass ihre Phantasien und Gefühle von der Konversation und dem Denken getrennt seien, aber sie war schon allzu fern, um meine Worte wirklich zu hören. Ich war «entthront» worden und hatte keine Möglichkeit mehr, ihre inneren Zustände zu regulieren. Niemand Neues kam, um diese Position zu besetzen und sie war noch nicht bereit, dies selber zu tun. Sie begann, auf der Beendigung der Therapie zu beharren, beschloss dann doch, zu bleiben, kam aber häufig zu spät oder sagte Sitzungen in letzter Minute ab. Es schien mir, dass wir mit unserer Arbeit noch nicht fertig waren und ich versuchte, ihre Trennungsängste zu deuten. Sie sagte mir, sie sei zu stur geworden, um noch etwas Neues von mir annehmen zu können. «Ihre Worte erreichen mich nicht», sagte sie einmal.

Sie erwähnte wieder Phantasien, viele Männer auf einmal zu haben, «vielleicht fünf Männer kommen sechs Mal in mich hinein.» Als sie wiederholte, die Wurzel dieses Wunsches sei ihr Gefühl von innerer Leere, hatte ich den Eindruck, es sei nichts Neues in ihrem Verständnis dazu gekommen. Ich versuchte, ihr zu sagen, dies könne zusammenhängen mit ihrer frühen Erfahrung von Hunger und Verlassenwerden, und die zahlreichen erigierten männlichen Penisse bedeuteten, dass sie jemandem wichtig war, dass sie es schaffte, jemanden zu erreichen. Ich versuchte ihr zu zeigen, dass ihre Absagen und ihre Gedanken an den Abschluss wahrscheinlich Bestrebungen waren, in mir das gleiche Gefühl von Vernachlässigung und Verlassenwerden hervorzurufen. Sie war damit einverstanden, aber alles ging auf die gleiche Weise weiter.

Sie bestand darauf, in völliger Unabhängigkeit weiterzuleben, und sie wisse ja, dass sie mich anrufen könne, wenn sie etwas bräuchte. Nach fast fünf Jahren wurde daher die Analyse beendet. Sie schien ein bisschen ängstlich, aber auch erleichtert. Ich äusserte meine Überlegungen und versuchte, eine gewisse Enttäuschung, so etwas wie: «Ich hätte gedacht, sie würde bis zum Ende kämpfen», zu verbergen. Aber was wäre das Ende, fragte ich mich. Ich konnte nur die Antwort finden, die ich bereits mit Frau A. geteilt hatte: eine neue Beziehung, anders als die vorherigen, mit der für ihre Erfahrung neuen Qualität der Verbindlichkeit.

Ich habe versucht, einige Fragmente von dem, was ich von Frau A. gelernt habe, zu verwenden, um das seltsame Schicksal des Konzepts Phantasie in der 
Psychoanalyse zu illustrieren. Ich bin zum Schluss gekommen, dass sowohl die übermässige Betonung als auch dieVernachlässigung der Bedeutung von Phantasie schädlich für die psychoanalytische Arbeit sind.

$\mathrm{Zu}$ Beginn ihrer Analyse zeigte Frau A. grosse Leidenschaft und viel Engagement für jedes Detail unserer Arbeit und unserer Beziehung. Sehr bald füllten sich die Sitzungen mit verschiedenen Ausdrucksformen der Erfahrung, dass ihre innere Welt nach mir verlangte, und gleichzeitig drang ich in sie ein und besetzte sie. Ich fühlte, dass sie abhängig wurde von mir, und dass meine Worte und Gesten ihre Emotionen immens beeinflussen konnten. Jedoch, egal wie viel sie über ihre sexuellen Phantasien sprach, erlebte ich diese nicht wirklich als sexuell. Alles, was Lehrbücher zu trennen versuchen, schien verstrickt und verwirrt. Auf einer Ebene, vielleicht der auffälligsten, schien Frau A. nicht in der Lage zu sein, zwischen Trieben und Fürsorge unterscheiden zu können, vor allem, wenn es um die Bedeutung des Vaters ging. Was sie beschrieb, könnte wie ödipale Phänomenologie ausgesehen haben, auch sagte sie: «Ich war in meinen Vater verliebt». Aber es stellte sich heraus, dass dies in ihrem verzweifelten Bedürfnis wurzelte, mit einer Figur zu verschmelzen, die für sie allwissend und allmächtig war. Manchmal reichte es, dass ein Mann nett zu ihr war (und ihr zum Beispiel bei der Bestellung von Meeresfrüchten half), dass Stunden später sexuelle Phantasien auftauchten. Auf einer tieferen Ebene war für mich sehr auffällig, wie die Motive Sexualität und Sicherheit - welche angeblich Freuds Modell von jenem Bowlbys unterscheiden bei Frau A. immer zusammen vorkamen, so dass sie nur eine Männerbeziehung einging, wenn sie annahm, dass der Mann in der Lage ist, sie zu beschützen, und sie konnte nur Lust empfinden mit Hilfe von Phantasien über viele Männer.

Der berührendste Teil dieser Analyse war unsere Arbeit an Frau A.s früher Verlassenheit, Vernachlässigung, und dem frühen Missbrauch. Diese schrecklichen Erfahrungen führten zu ihrem übermässigen Bedürfnis nach Bindung und ihrer Unfähigkeit, über diese Bedürfnisse nachzudenken (d.h. sie zu mentalisieren) und nach Wegen zu suchen, sie zu befriedigen. Es war ihre traumatische Kindheit, welche sie später zu ungewöhnlichem sexuellen Verhalten und zu noch ungewöhnlicheren sexuellen Phantasien führte, die in stark verhüllter Form ihr Erleben von innerer Leere und Wertlosigkeit ausdrückten. Dies führte sie immer wieder von Neuem zu einer retraumatisierenden Partnerwahl und zu noch seltsameren Phantasien und agierenden Verhaltensweisen, die ihr Leben wiederholt in einen Teufelskreis führten.

Ich kann nur hoffen, dass die beschriebene Fallstudie zeigt, dass Psychoanalyse nicht weniger offen für neue Erfahrungen und Konzepte sein soll, als dies 
ihre Klienten sind. Wenn Psychoanalyse im einundzwanzigsten Jahrhundert relevant bleiben will, muss sie sich auf die zeitgenössische Wissenschaft, Gesellschaft und heutigen Krankheitsbilder einstellen; und wenn Bindungstheorie und Forschung für die Psychoanalyse an Bedeutung gewinnen sollen, muss die innere Welt der Patienten gründlicher erforscht werden. Die Psychoanalyse hätte nie vor die Entscheidung gestellt werden sollen, zwischen dem Trauma-Modell und dem Phantasie-Modell wählen zu müssen, weil die Phänomene, auf welche sich diese Modelle beziehen, klinisch nicht getrennt werden können.

\section{Literatur}

Bateman, Anthony; Peter Fonagy und John G. Allen (2009): Theory and Practice of Mentalization-Based Therapy. In: Glen O. Gabbard, Hrsg., Textbook of Psychotherapeutic Treatments, Washington, D.C.: American Psychiatric Publishing, 757-780.

Bergmann, Martin S. (2004): Conference Proceedings. In: Martin S. Bergmann, Hrsg., Understanding Dissidence and Controversy in the History of Psychoanalysis, New York: Other Press, 259-358.

Bowlby, John (1965): Child Care and the Growth of Love. London: Penguin Books. Bowlby, John (1969): Attachment. Attachment and Loss (vol. 1). London: Hogarth Press.

Bowlby, John (1973): Separation: Anxiety \& Anger. Attachment and Loss (vol. 2). London: Hogarth Press.

Bowlby, John (1980): Loss: Sadness \& Depression. Attachment and Loss (vol. 3). London: Hogarth Press.

Cassidy, Jude und Philip R. Shaver (Hrsg.) (2008): Handbook of Attachment. Theory, Research, and Clinical Applications. NewYork-London: The Guilford Press. Fonagy, Peter (2003): Bindungstheorie und Psychoanalyse. Stuttgart: Klett-Cotta. Fonagy, Peter und Anthony Bateman (2008): Mentalization-Based Treatment of Borderline Personality Disorder. In: Elliot L. Jurist; Arietta Slade und Sharone Bergner, Hrsg., Mind to Mind. Infant Research, Neuroscience, and Psychoanalysis, New York: Other Press, 139-166.

Issroff, Judith (2005): Donald Winnicott and John Bowlby. Personal and Professional Perspectives. London - New York: Karnac.

Kahr, Brett (2007): Sex and the Psyche. The Truth about Our Most Secret Fantasies. London: Penguin Books. [Dt.: Kahr, Brett (2008): Sex im Kopf: Alles über unsere geheimsten Fantasien. Berlin: Ullstein.] 
Masson, Jeffrey Mousaieff (1985): The Assault on Truth. Freud's Suppression of the Seduction Theory. New York: Farrar, Straus \& Giroux.

Rudnytsky, Peter L. (2000): Psychoanalytic Conversations. Interviews with Clinicians, Commentators, and Critics. Hillsdale, NJ - London: The Analytic Press.

Stepansky, Paul E. (2009): Psychoanalysis at the Margins. New York: Other Press.

Winnicott, Donald W. ([1959] 1989): Discussion of Mourning and Grief in Infancy, by John Bowlby. In: Clare Winnicott, Ray Shepherd und Madeleine Davis, Hrsg., Psychoanalytic Explorations, London: Karnac, 426-432.

\section{Anmerkung}

1 Aus dem Englischen übersetzt von Maria Haag Turner und Regula Schiess. 